\section{$\underset{\substack{\text { hommes } \\ \text { \& migrations }}}{ }$}

\section{Hommes \& migrations}

Revue française de référence sur les dynamiques

migratoires

$1315 \mid 2016$

Ondes de choc

\title{
L'attentat contre Charlie Hebdo vu du Québec
}

Une discussion sur la liberté d'expression et la critique de la religion

\section{Gilles Gauthier}

\section{(e) OpenEdition \\ 1 Journals}

\section{Édition électronique}

URL : http://journals.openedition.org/hommesmigrations/3712

DOI : 10.4000/hommesmigrations.3712

ISSN : 2262-3353

\section{Éditeur}

Musée national de l'histoire de l'immigration

\section{Édition imprimée}

Date de publication : 1 juillet 2016

Pagination : $33-41$

ISBN : 978-2-919040-36-0

ISSN : $1142-852 X$

\section{Référence électronique}

Gilles Gauthier, «L'attentat contre Charlie Hebdo vu du Québec », Hommes \& migrations [En ligne], 1315 | 2016, mis en ligne le 01 juillet 2019, consulté le 02 janvier 2020. URL : http://journals.openedition.org/ hommesmigrations/3712; DOI : 10.4000/hommesmigrations.3712 


\title{
L'ATTENTAT CONTRE CHARLIE HEBDO VU DU QUÉBEC \\ UNE DISCUSSION SUR LA LIBERTÉ D'EXPRESSION ET LA CRITIQUE DE LA RELIGION
}

Par GILLES GAUTHIER, professeur au Département d'information et de communication de l'université de Laval (Québec).

\author{
L'attentat commis contre le magazine satirique \\ Charlie Hebdo le 7 janvier 2015 à Paris a bouleversé la presse \\ internationale. Entre les messages de soutien affluant \\ du monde entier se diffuse également le besoin d'analyser \\ la situation, de comprendre l'origine de la menace \\ et au nom de quoi des journalistes ont pu ainsi être assassinés. \\ Au Québec, dès les jours qui suivent l'événement, le débat \\ médiatique se focalise sur le lien problématique entre défense \\ de la liberté d'expression et critique des religions. \\ Or les journalistes qui y prennent part sont loin d'être unanimes \\ et obéissent chacun à des présupposés différents.
}

$\mathrm{Au}$ Québec, comme partout ailleurs dans le monde occidental, l'une des réactions très fortes à l'attentat commis contre Charlie Hebdo en janvier 2015 a consisté en une défense de la liberté d'expression. Parce que l'acte terroriste avait été commis contre un magazine satirique qui, précisément, défiait toute forme de censure, il était normal de le condamner au nom de la liberté de pensée et de parole. Cette apologie de la liberté d'expression s'est immédiatement portée sur un droit de critiquer la religion. La chose nétait pas non plus étonnante dans la mesure où le motif reconnu de l'attentat a été l'attitude provocatrice affichée par l'hebdomadaire à l'égard des religions, tout spécialement l'islam, par la publication des caricatures de Mahomet.

Telle fut la ligne de pensée cardinale exprimée au lendemain de la tuerie. Mais, au-delà de la liaison formelle faite entre la sauvegarde de la liberté d'expression et le droit de critiquer la religion, les 


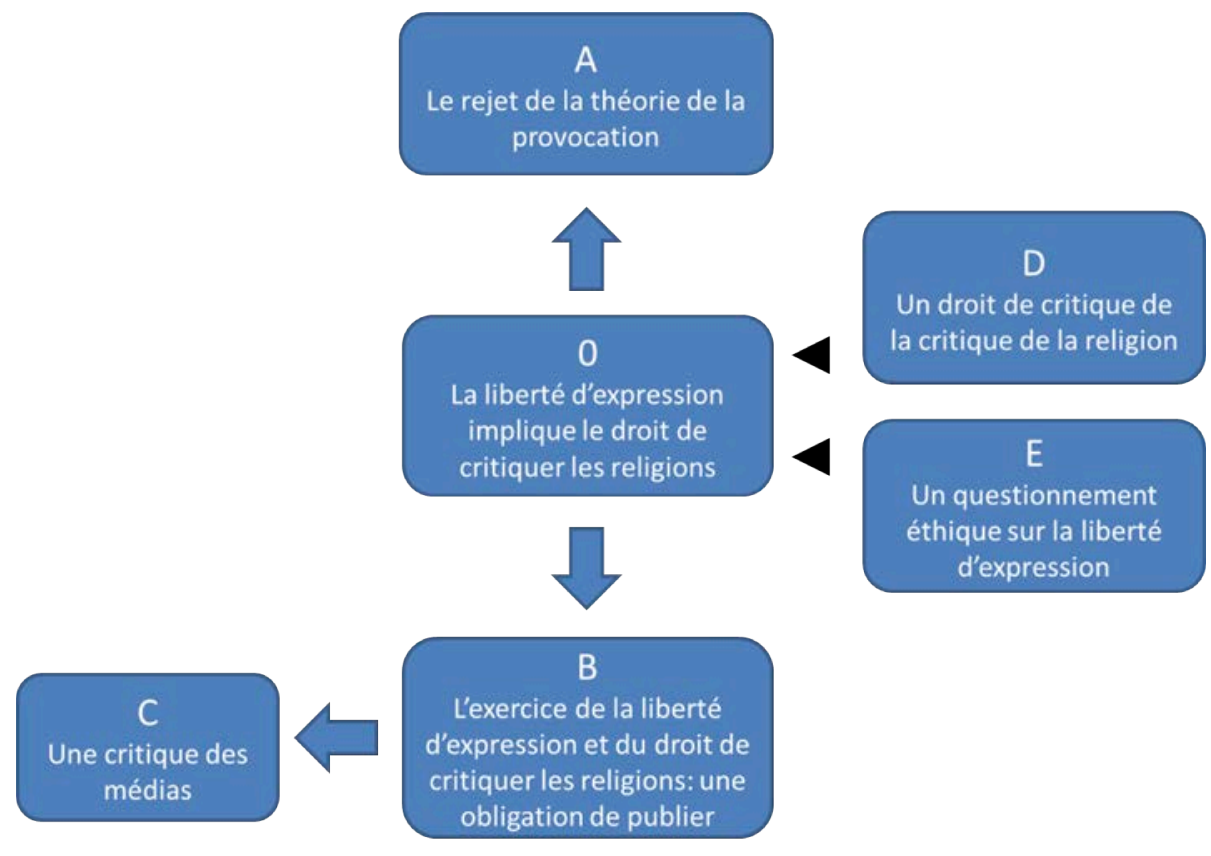

positions prises sur les implications précises de cette relation, ainsi que sur l'exercice par Charlie Hebdo et l'ensemble des médias de leur liberté d'expression n'ont pas été consensuelles. Elles ont, au contraire, donné lieu à des clivages qui ont fait se déployer la discussion sur des enjeux connexes et pour certains plus complexes.

Je me donne ici pour objectif d'étudier cette expansion du débat. Je veux rendre compte des principaux éléments en fonction desquels les éditorialistes et les chroniqueurs de la presse québécoise francophone, ainsi que d'autres intervenants ayant exprimé leur point de vue dans les médias traditionnels ou numériques ont analysé plus finement et plus systématiquement la relation entre la liberté d'expression et le droit de critiquer la reli- gion. Plus précisément, je m'efforcerai de saisir les considérations diverses en fonction desquelles est comprise différemment la possibilité de ridiculiser ou même de dénigrer les religions que tous reconnaissent découler du principe de la liberté d'expression.

\section{L'idée d'amplitude du débat public}

Un débat n'est pas statique. Les désaccords sur lesquels il porte, ainsi que les interactions sociales dont il procède et qu'il met en marche bougent, et ces modifications viennent bousculer son état d'équilibre provisoire et réorganiser sa structu- 
ration. Deux composantes distinctes de la dynamique du débat public peuvent être identifiées. La première est sa mobilité ; elle concerne les modifications que peut connaître un débat ou les mutations d'enjeux qui le métamorphosent à travers le temps. L'autre est son amplitude ${ }^{1}$. Considérés en coupe synchronique, les paramètres d'un débat ne sont pas fixes et rigides mais plutôt mouvants. Il y a un mouvement intérieur à un débat, différent de celui de son évolution historique, qui fait que sa configuration bouge au gré des accentuations, déviations, altérations et autres mouvements produits par les différentes interventions auxquelles il donne lieu. L'amplitude d'un débat, c'est cette oscillation, cette élasticité qui le fait respirer. C'est suivant son amplitude que l'extension d'un débat peut se voir corriger. Il peut se dilater quand ses tenants et aboutissants s'élargissent ou, au contraire, se contracter, quand ils se rétrécissent.

\section{Une représentation schématique}

L'amplitude du débat sur la liberté d'expression et le droit de critiquer la religion tel qu'il s'est tenu au Québec après l'attentat contre Charlie Hebdo peut être représentée par le schéma suivant. À partir de son nœud central posant que la liberté d'expression implique le droit de critiquer la religion (0), certains justifient le rejet de la thèse de la provocation par Charlie Hebdo (A) ; d'autres interprètent le droit de critiquer les religions découlant de la liberté d'expression comme une obligation (B)... en vertu de laquelle ils font reproche aux médias d'avoir plié face à la menace terroriste et à l'islamisme en ne publiant pas les caricatures de Mahomet (C) ; d'autres encore font valoir que la critique de la religion revendiquée comme un droit afférent à la liberté d'expression peut elle-même être critiquée (D) ; dans sa forme la plus achevée, cette idée culmine dans une discussion éthique sur la liberté d'expression et le droit de critiquer les religions, ainsi que sur leur exercice par Charlie Hebdo (E).

A, B, C, D et E constituent les pôles autour desquels s'articulent les interventions qui impriment les différents mouvements synchroniques dont est imprégné le débat et qui marquent son déploiement multiaxial. Comme nous l'analyserons en détail, ces points pivots sont d'une importance inégale et n'influent pas sur le débat d'une manière uniforme. Ils n'entretiennent pas non plus le même type de rap-
Tous les éditorialistes et chroniqueurs qui commentent la tuerie à Charlie Hebdo la condamnent au nom du principe de la liberté d'expression, en le mettant en rapport avec le droit de critiquer la religion. port avec son point nodal.

Comme le fait voir le schéma, $A$, B et $C$ sont induits de la relation entre la liberté d'expression et le droit de critiquer la religion, alors que $\mathrm{D}$ et $\mathrm{E}$ constituent plutôt des réactions à ce rapport (les deux liens sont exprimés dans le schéma respectivement par les symboles et $\mathbf{-}$ ).

\section{La possibilité de critiquer la religion (0)}

Tous les éditorialistes et chroniqueurs qui commentent la tuerie à Charlie Hebdo la condamnent au nom du principe de la liberté d'expression, en le mettant en rapport avec le droit de critiquer la religion. Certains le font de manière implicite en endossant l'attitude du magazine à l'égard de l'islamisme : "Pourquoi un hebdomadaire satirique suscitet-il autant de haine? Parce que la caricature expose, mieux que tout autre procédé, les failles dans l'édifice de l'islamisme radical, l'absurdité et la bêtise qui servent d'idéologie aux fondamentalistes, aveuglés par leur croyance. (...) [Les] dessins [des caricaturistes] ne sont par une provocation gratuite. Ils affirment d'abord et avant tout la liberté de pensée et de parole². " D'autres établissent le lien en formulant la réponse à donner à l'attentat: "Il faut s'obliger, et il y a urgence, à 
garder les fenêtres ouvertes, que les idées circulent, que chacun trouve son ton, mais que les débats se fassent, que les opinions se confrontent et provoquent, surtout en matière religieuse, lieu des nouveaux tabous ${ }^{3}$. "

D'autres encore font valoir que le droit de critique des religions est caractéristique de la France : «En s'attaquant à Charlie Hebdo, les islamistes s'attaquaient à l'esprit même de ce pays... où il est permis de rire de tout. (...) en France, le droit de critiquer les religions, comme n'importe quelle autre idéologie
L'idée que Charlie Hebdo

ait été l'artisan de son

propre malheur en osant

publier les caricatures

de Mahomet est repoussée

par tous les intervenants qui

se prononcent à ce sujet. d'ailleurs, est inscrit non seulement dans la loi mais dans l'histoire 4 ."

Le lien entre la liberté d'expression et le droit de critiquer la religion est posé comme un principe théorique par certains. C'est notamment le cas du texte signé par l'ensemble de l'équipe éditoriale de La Presse pour laquelle la « liberté d'expression des satiristes » n'est en fait qu'un prolongement de "notre droit à nous, citoyens, d'exprimer notre opinion sur l'islam... que cette opinion soit modérément ou farouchement critique, qu'elle se décline en mots ou en images ${ }^{5}$ ». Aussi, selon ces éditorialistes, le droit de critiquer les religions est le contrepoids la liberté de religion : "dans une société démocratique, [le] droit [des croyants d'exprimer leur foi sur la place publique] à une contrepartie : la liberté d'exprimer son opinion sur les religions, d'en contester la véracité, de dénoncer l'exploitation qui en est faite, et aussi de s'en moquer ${ }^{6}$ ».

\section{Le rejet de la théorie de la provocation (A)}

L'idée que Charlie Hebdo ait été l'artisan de son propre malheur en osant publier les caricatures de Mahomet est repoussée par tous les intervenants qui se prononcent à ce sujet. Certains le font pour une raison circonstancielle : "Certains se sont demandé si les journalistes de Charlie Hebdo ne porteraient pas une part de responsabilité dans ce qui vient d'arriver. Il me semble déplacé de poser cette question en ce moment. Quoi qu'on en pense, il y a une disproportion choquante entre le style provocateur quion pourrait reprocher au journal et la violence de l'attaque ${ }^{7}$. " Le plus souvent, cependant, la thèse de la provocation est désavouée au motif que le droit de critiquer les religions découle de la reconnaissance de la liberté d'expression : " “Oui, mais". C'est ce qu'ont répété plusieurs critiques de Charlie Hebdo au cours des dernières années. Certes, la liberté d'expression des satiristes doit être protégée, répétait-on unanimement. Mais, ajoutaient certains, il fallait éviter de "provoquer" les terroristes. Ces bémols, quion a continué à entendre hier, sont infondés et dangereux. (...) Bien sûr qu'il y a un lien de cause à effet entre les caricatures et l'attentat. C'est une évidence! Mais cela ne rend d'aucune façon la victime responsable. L'affirmer serait prétendre que la violence intégriste constitue une réponse légitime à une critique de la religion. Et donc qu'un satiriste ne serait pas une victime innocente. La thèse de la provocation trahit une capitulation intellectuelle. Comme s'il existait un droit à ne pas être insulté, une interdiction de blasphème ${ }^{8}$. »

\section{La liberté d'expression: du droit à l'obligation (B)}

Dans la foulée du rejet de la théorie de la provocation, des chroniqueurs vont jusqu'à affirmer que le principe de la liberté d'expression exigeait la publication des caricatures de Mahomet. C'est le cas, par exemple, de Josée Legault qui considère que la décision de ne pas le faire constitue un acte d'autocensure. La justification qu'elle offre à ce propos est d'ordre politique : "[Le] message est, en fait, que la peur protège des intégristes. Alors qu'heureusement on peut représenter et rire sans crainte des figures chrétiennes ou juives, il donne aux leaders musulmans l'immense pouvoir de juger de ce qui est ou non une limite 
raisonnable à la liberté d'expression dans une société démocratique. Ce faisant, il le retire aux parlements et aux lois. Cette censure sélective trahit aussi un glissement sur le concept même de religion. En prêtant à Charlie Hebdo l'intention "d'offenser" les musulmans, elle confond l'islam et la vraie cible du magazine : les intégristes religieux de tout acabit ${ }^{9}$. "

Rima Elkouri adopte une position allant dans le même sens en exprimant l'avis que les médias se devaient, après l'attentat, de publier les caricatures de Mahomet : «À mon sens, par solidarité, il fallait publier les caricatures de Charlie Hebdo comme l'ont fait de nombreux médias d'ici et d'ailleurs. Il fallait le faire à la mémoire des victimes. Il fallait le faire pour réaffirmer notre attachement à ce principe fondamental qu'est la liberté d'expression ${ }^{10}$. » Pierre Trudel s'inscrit en faux contre cette représentation de la liberté d'expression comme un devoir plutôt que comme un droit. À ses yeux, "le droit de publier inclut aussi le droit de ne pas publier. (...) Dans les sociétés démocratiques, les médias sont titulaires de la liberté de pensée et d'expression. Tous peuvent avoir leur opinion sur l'opportunité de diffuser tel ou tel contenu. La liberté d'expression et de la presse garantit et protège le droit de choisir de publier ou de ne pas publier une information. C'est ce qu'on appelle la liberté éditoriale, celle qui garantit à tout média le droit de choisir ce quill publie, de sélectionner ce qu'il va diffuser ou son droit de ne pas publier ${ }^{11}$. »

\section{Pour une critique des médias (c)}

Parmi ceux qui considèrent la liberté d'expression comme un devoir, certains comme Josée Legault reprochent aux médias d'avoir succombé à la peur et de manquer de courage face à l'islamisme : «Si Charlie Hebdo est devenu une cible, c'est parce qu'en Occident, d'autres médias ont préféré céder à la peur de tenir tête. (...) L'incapacité de ces médias à comprendre l'urgence de poser ce geste, même tardif, pour préser- ver leur propre liberté de pensée est sidérante. C'est à croire qu'ils en ont déjà fait leur deuil. Pire encore, en refusant ce geste de solidarité envers les morts de Charlie Hebdo, ces médias donnent en fait raison aux intégristes. La peur que ce refus trahit est leur récompens $e^{12}$. » Bien qu'il refuse d'inférer une obligation de publier du principe de la liberté d'expression, Pierre Trudel admet, par ailleurs, que son exercice par les médias peut être critiqué : "Chacun peut évidemment manifester son désaccord avec les choix rédactionnels d'un média ${ }^{13}$. » Richard Martineau s'exclame: "Honte aux journaux qui, comme l'ont fait The Guardian et le New York Daily News, "brouillent" les couvertures de Charlie Hebdo pour ne pas offenser certains de leurs lecteurs ${ }^{14}$. "

\section{Le droit à la critique de la critique de la religion (D)}

Certains, une fois admis que le droit de critiquer les religions découle de la liberté d'expression, revendiquent, comme Rima Elkouri, le même droit à l'égard de cette critique elle-même : "Dans les sociétés où la liberté d'expression veut dire quelque chose, on ne peut remettre en question le droit d'un Parailleurs, la reconnaissance journal de publier les caricatures qui lui plaisent, même (ou surtout) si cela déplaît. En démocratie, on doit toutefois pouvoir en discuter et en débattre. Car la liberté d'expression, c'est aussi la d'un droit de critique de la critique médiatique de la religion débouche également sur une discussion particulièrement développée sur la liberté d'expression d'un point de vue éthique. liberté d'exercer son jugement critique ${ }^{15}$. " Ainsi conçu, le droit de critique de la critique des religions ne repose pas sur une exigence éthique. Il est simplement vu comme une extension de la liberté d'expression qui loin d'être un contrepoids qui en restreindrait la portée tout au contraire l'élargit.

9. Josée Legault, «Blâmer la victime ", in Le Journal de Québec, 12 janvier 2015. 10. Rima Elkouri, " Endeuillés et meurtris ", in La Presse, 9 janvier 2015. 11. Pierre Trudel, "Les médias ont le droit de s'autocensurer ", in Le Journal de Québec, 10 janvier 2015. 12. Josée Legault, «La peur n'est pas une option », in Le Journal de Québec, 8 janvier 2015. 13. Pierre Trudel, "Les médias ont le droit de s'autocensurer ", op. cit. 14. Richard Martineau, "L’attentat de trop ", in Le Journal de Québec, 8 janvier 2015 . 


\section{Un questionnement éthique $(E)$}

Par ailleurs, la reconnaissance d'un droit de critique de la critique médiatique de la religion débouche également sur une discussion particulièrement développée sur la liberté d'expression d'un point de vue éthique. Ce sont surtout des intellectuels, des philosophes et des juristes qui impriment ce tournant au débat. Jocelyn Maclure commence par s'insurger contre ce qu'il appelle une « censure inversée » qui interdirait de blâmer Charlie Hebdo : "Si le droit

Cette considération éthique de la liberté d'expression ainsi que de son exercice par Charlie

Hebdo et par l'ensemble des médias suscitent deux réactions

distinctes: l'une s'y oppose en la discutant pour

elle-même; l'autre la rejette plus radicalement en excluant

tout rapport entre éthique et liberté d'expression. de critiquer les religions doit être défendu, celui d'en découdre avec ces critiques doit l'être également ${ }^{16}$." Maclure s'autorise, ensuite, à exercer ce droit de critique de la critique de la religion en formulant un jugement moral, qu'il distingue d'un motif juridique, sur Charlie Hebdo:

"La publication des caricatures me semblait défendable d'un point de vue juridique, mais déplorable d'un point de vue éthique. Ce n'est pas parce qu'on a le droit de publier quelque chose qu'on doit le faire ${ }^{17}$. " Le philosophe appuie cette estimation éthique sur deux justifications complémentaires : le fait que les musulmans se sentent insultés par la publication des caricatures et que celle-ci détériore le débat public : «En l'occurrence, publier les caricatures qu'un grand nombre de musulmans allaient juger injurieuses dans un contexte marqué par l'islamophobie post11 septembre, cela manquait fortement de sagesse et d'empathie ${ }^{18}$. " Maclure écrit aussi : " [Les caricaturistes de Charlie Hebdo ont manqué] de jugement éthique en publiant des contenus incen- diaires contribuant de façon médiocre à la qualité du débat ${ }^{19}$. " Dans une perspective apparentée, mais sans cependant exprimer un blâme moral aussi explicite, Jean-Pierre Proulx propose quelques balises d'une «éthique journalistique de la liberté d'expression ». Il spécifie d'abord qu'elle n'est pas absolue, mais assujettie (ainsi que le pose le préambule de la Charte québécoise des droits et libertés de la personne) au " "respect de la dignité de l'être humain et [à] la reconnaissance des droits et libertés dont il est l'objet [qui] constituent le fondement de la justice et de la paix"20 ". Après avoir ensuite rappelé qu'elle est déjà juridiquement limitée, Proulx avance que, comme toutes les autres libertés, la liberté d'expression est « au quotidien... avant tout régulée par l'éthique [qui] donne sens à nos actions parce qu'elle repose sur nos valeurs (...) et passe aussi par les vertus ${ }^{21}$ ". Proulx invoque à ce double propos la valeur du respect et la vertu de la prudence puis, par la suite, la nécessité de tenir compte de la réception des messages et d'assortir la force des moyens d'expression (la moquerie, la satire, la caricature, la provocation) à l'importance des enjeux. Au total, au sujet de la publication des caricatures de Mahomet, Proulx, tout en reconnaissant que ce "jugement prudentiel... est... fort difficile à poser ", émet l'avis suivant: «La prudence peut aussi mener à conclure qu'il vaut mieux s'abstenir s'il apparaît que les conséquences de la prise de parole seront plus dommageables que l'abstention. Ainsi, les médias anglophones, contrairement aux francophones, ont jugé préférable de ne pas publier les caricatures de Charlie Hebdo ${ }^{22}$. » Pour certains, c'était là donner raison aux terroristes. Je préfère penser que chacun a fait un choix prudentiel différent. Il conclut, sans toutefois évoquer dans un sens ou dans l'autre Charlie Hebdo, en stipulant: «celui qui s'exprime ne peut être volontairement insultant, car c'est porter atteinte à la dignité de celui ou de ceux à qui il s'adresse. C'est éthiquement la limite à ne pas franchir ${ }^{23}$ ». 


\section{La liberté d'offenser}

Cette considération éthique de la liberté d'expression ainsi que de son exercice par Charlie Hebdo et par l'ensemble des médias suscitent deux réactions distinctes : l'une s'y oppose en la discutant pour elle-même ; l'autre la rejette plus radicalement en excluant tout rapport entre éthique et liberté d'expression. Daniel Weinstock réfute la réprobation morale de la publication des caricatures de Mahomet par Charlie Hebdo exprimée par Maclure et Proulx, d'abord en faisant valoir que, en démocratie, s'il existe un droit à ne pas être diffamé, il n'y en a pas à ne pas être visé par un discours haineux et à ne pas être injurié et que, en conséquence, il n'y a pas obligation à se censurer de peur d'offenser, mais plutôt une obligation de supporter cette offense : "Une obligation s'impose à ceux dont les croyances religieuses peuvent avoir été heurtées d'avoir la capacité de le supporter et non pas une obligation de se censurer à ceux qui les ont offensés ${ }^{24}$. " Ainsi, Weinstock module ce principe théorique en fonction de considérations éthiques plus concrètes relevant du vivre ensemble. Il importe, selon lui, de tenir compte du rapport hiérarchique entre offenseurs et offensés, ainsi que de la relation de confiance ou de méfiance qui existe entre eux. Il lui semble, à ce propos, que la précarité de la situation des musulmans dans les sociétés occidentales fait en sorte que n'y est pas établi avec eux un rapport de confiance et d'égalité, ce qui rend compréhensible qu'ils aient le sentiment que les caricatures de Charlie Hebdo renforcent leur marginalisation, qu'ils se sentent heurter et qu'il leur soit difficile de se plier à l'exigence de supporter l'offense faite à leurs croyances religieuses.

Weinstock n'en tire pas la conclusion que la publication des caricatures aie été moralement répréhensible. Pour le déterminer, il propose de considérer deux interprétations possibles de l'intention de Charlie Hebdo de ridiculiser l'islam en publiant les caricatures. Suivant la première interprétation, l'hebdomadaire satirique cherchait uniquement à choquer et à offenser les musulmans ; suivant la seconde, l'objectif poursuivi était de faire valoir, sous une forme choquante, quelque idée à propos des croyances et des symboles religieux de l'islam. Cela procédait donc d'une visée de communication. Considérant que c'est cette dernière interprétation qui prévaut, Weinstock avance que la publication des caricatures relevait d'un droit moral et non seulement légal, peu importe qu'elle apparaisse potentiellement destructrice du lien social : «S'il y a une vraie intention de communication que ne contrecarrent pas les dispositions légales relatives au discours haineux, à l'incitation à la violence, au libelle et à la diffamation et autres, ma position est que nous devons reconnaître non seulement un droit légal, mais aussi un droit moral de défendre des points de vue même si nous nous efforçons de monter quills sont inappropriés ${ }^{25}$." Il faut ainsi distinguer entre considérer que le propos de quelqu'un est moralement répréhensible et considérer que cette personne est moralement répréhensible de tenir ce propos que nous tenons comme moralement répréhensible : "C'est une chose d'affirmer qu'un propos est moralement répréhensible; c'en est une autre de prétendre que la personne qui le tient a moralement tort de tenir ce propos que nous jugeons moralement répréhensible ${ }^{26}$. »

Au total, selon Weinstock, la considération morale de la publication des caricatures de Mahomet mène à une situation inextricable. Nous devons admettre que les caricaturistes de Charlie Hebdo étaient éthiquement justifiés à formuler leur point de vue de la façon qu'ils ont choisi de le faire, tout en reconnaissant que les musulmans pouvaient légitimement se sentir offensés et que leur sentiment de marginalisation s'en trouvait exacerbé. Face à ce cassetête, Weinstock préconise deux choses : combattre les causes de l'exclusion sociale qui rendent difficile pour les musulmans de tolérer d'être offensés et de sentir qu'ils ont leur place dans les sociétés occidentales, et s'assurer que le droit à la liberté d'expression, y compris dans sa possibilité de communiquer des idées de manière choquante, soit étendu à tous les groupes. En formulant cette conclusion, Weinstock engage le débat dans une nouvelle dimension: 
il le fait passer de la question de la liberté d'expression et du droit de critiquer la religion à celle, beaucoup plus vaste, de l'organisation sociale. Ce faisant, il établit un lien (resté implicite chez Maclure) entre éthique et philosophie politique ${ }^{27}$.

\section{Expression du droit et vision du monde}

Alors que Weinstock réfute l'appréciation morale de la liberté d'expression de Maclure et Proulx en demeurant dans une perspective éthique, Antoine Robitaille et Pierre Trudel contestent la possibilité même d'en apprécier l'exercice et de l'encadrer à partir d'un point de vue éthique. Robitaille met en question la limite éthique que certains voudraient voir imposer à la liberté d'expression de manière concise : "Une mouvance tente de trouver un moyen terme entre la légalité (en France, la republication de ces dessins était permise) et une position "éthique" de retenue éclairée. Le droit permet cette publication. Le sens éthique aurait dû l'empêcher. Un droit qu'il est préférable de ne pas exercer est-il encore un droit? Le droit au blasphème, à la caricature, qui n'offenserait jamais personne n'est-il pas contradictoire dans les termes. La liberté d'expression "dans le respect"... est-ce une chose concevable $^{28}$ ? " Trudel, quant à lui, développe une argumentation plus étendue. Sa thèse centrale est que la liberté d'expression ne peut être régulée que par le droit : "Il faut cesser de préconiser de limiter les libertés expressives au-delà des seules limites imposées par des lois dont la raisonnabilité est vérifiée par des juges indépendants ${ }^{29}$. "Selon lui, invoquer des droits religieux et, plus généralement, des "références "culturelles" [ou] "religieuses" » tend à réduire la liberté d'expression et, du même coup, à étouffer le débat public et à donner raison à l'obscurantisme : "À force de répéter qu'il faut éviter de "choquer", d'indisposer, de déranger lorsqu'on caricature et critique, certains chantres de "l'éthique" de l'information contribuent à rapetisser l'espace de la liberté d'expression. (...) À vouloir protéger les droits constamment au prix de limites toujours accrues à la liberté d'expression, l'on contribue à taire les débats, supprimer les discussions ${ }^{30}$. » Pour Trudel, un trop grand respect des " "sensibilités" culturelles » qui conduit à la censure a l'effet pervers de donner raison à l'obscurantisme. À ses yeux, tout au contraire, c'est en ouvrant le débat, en permettant encore plus de prise de parole que l'on assure le respect des droits de toutes et de tous, pas en les muselant ${ }^{31}$. C'est d'ailleurs à l'encontre de «ceux qui discourent sur léthique et la "responsabilité" de ceux qui s'expriment » que Trudel s'oppose, comme rapporté plus haut, à l'idée de la liberté d'expression comme un devoir et revendique pour le droit de publier un droit de ne pas publier. "Cette faculté que possèdent les médias de faire des choix éditoriaux différents en fonction de leurs croyances, leurs évaluations, leurs valeurs... [est] inhérente à la liberté d'expression. Elle est garante du pluralisme des idées et des opinions ${ }^{32}$. " Trudel s'attaque de la sorte à un certain point de vue éthique sur la liberté d'expression : "Il [serait] dangereux de se mettre à réclamer que les évaluations fondées sur les différentes "éthiques" qui coexistent dans les sociétés démocratiques deviennent autant de balises obligatoires pour les médias. (...) Les évaluations que font les médias de l'intérêt public, de ce qui est nécessaire ou opportun de montrer ou non reflètent leurs conceptions et visions du monde. Il est normal et sain que ces conceptions et visions diffèrent d'un média à l'autre. Les discours se réclamant des différentes "éthiques" de linformation, les discours religieux, les évaluations morales de tous ceux qui sarrogent le droit de juger le comportement "éthique" des autres, appartiennent au domaine des idées. Chacun est libre de les partager ou $n n^{33}$. " Selon Trudel, le pluralisme des valeurs au cœur de l'éthique interdit que cette dernière vienne contraindre la liberté d'expression et ainsi mettre en échec le pluralisme de l'information : "Lorsquion prétend, au nom de certaines conceptions de "l'éthique", imposer des façons de voir à tous, il y a danger pour la liberté de presse et le pluralisme de l'information ${ }^{34}$. " 


\section{Conclusion}

À la vue de l'analyse de l'amplitude du débat suscité au Québec par la relation entre liberté d'expression et droit de critiquer la religion, certains points saillants méritent d'être relevés. L'examen de la modulation du débat met clairement en évidence la prépondérance du pôle E. La discussion sur les autres pôles s'épuise assez rapidement alors que celle sur le questionnement éthique de la liberté d'expression et du droit de critiquer la religion gagne en ampleur au fur et à mesure des interventions. Sa prééminence est telle que la discussion publique que suscite l'attentat contre Charlie Hebdo finit par se focaliser sur les questions de savoir si et comment la liberté d'expression peut être balisée et limitée par des considérations d'ordre moral. L'affrontement décisif qui naît du mouvement d'amplitude qui agite le débat met aux prises ceux qui le prétendent, comme Maclure, Proulx et Weinstock, et ceux qui récusent toute mainmise ou saisie éthique, comme Robitaille et Trudel. Deux aspects généraux ressortent de leur désaccord. Le premier a trait à la relation entre l'éthique et le droit ou, plus précisément, à l'impact des deux modes de régulation sur la liberté d'expression. Pour Maclure, Proulx et Weinstock, le fait que la liberté d'expression soit soumise à certaines contraintes légales n'empêche pas qu'elle puisse aussi faire l'objet d'une évaluation morale. Pour Robitaille et Trudel, au contraire, l'encadrement légal de la liberté d'expression est suffisant et exclut la pertinence de toute appréciation éthique. Par ailleurs, la mésentente sur la possibilité même de considérer la liberté d'expression d'un point de vue moral repose sur une opposition entre deux conceptions de l'éthique. Pour Trudel (la chose n'est pas claire dans le cas de Robitaille étant donné la concision de son propos), qui met le plus souvent le terme entre guillemets, « léthique » relève de la subjectivité de chacun. Elle est affaire «[de] croyances, [d']évaluations, [de] valeurs » que " chacun est libre de partager... ou non ». L'éthique, essentiellement personnelle, est donc plurielle : il y a des «éthiques », c'est-à-dire une diversité de systèmes moraux. C'est très précisément afin de prémunir la liberté d'expression contre la prédominance possible d'un de ces systèmes moraux qui contraindrait le débat public que Trudel s'insurge contre toute évaluation éthique. Àses yeux, il faut tenirl'éthiqueà distance de la liberté d'expression pour permettre le pluralisme des idées, des opinions et de l'information. Ceux qui, au contraire, conçoivent que la liberté d'expression peut être un objet d'examen pour l'éthique en ont une conception plus objectivante. Sans nier que les valeurs morales relèvent d'une adhésion personnelle, ils considèrent qu'elles peuvent donner lieu à une réflexion rationnelle. Maclure réfère à l'« empathie»; Proulx au « respect de la dignité de l'être humain » et à la "prudence»; Weinstock, au « rapport de confiance " ainsi qu'à l'« égalité », et tous les trois à la qualité des rapports sociaux. C'est précisément en prétendant mener une analyse raisonnée qu'ils cherchent à déterminer comment la liberté d'expression est assujettie à l'éthique ${ }^{35}$.

Le questionnement éthique produit par la réaction à l'attentat contre Charlie Hebdo ne lui est pas spécifique. Le débat public contemporain, en tout cas au Québec, fait l'objet d'une forte moralisation : la discussion sur les enjeux sociaux, qu'ils portent sur des questions en elles-mêmes morales ou non, est marquée par une préoccupation éthique notable ${ }^{36}$. Cette incidence éthique se manifeste, entre autres choses, par l'usage abondant de justifications morales : très souvent, on fait reposer les positions adoptées dans les débats sur des fondements normatif $s^{37}$. Le recours à l'argumentation morale est d'ailleurs l'un des principaux mécanismes de majoration d'amplitude du débat public ${ }^{38}$. 